Reveiw Article

Yam Bahadur Roka

MS, MCh., IFAANS

Dr. Yam Bahadur Roka

M.S. (Surgery), M.Ch. (Neurosurgery), F.N.N.I. (Skull

Base Surgery), IFAANS

Chief of Neurosurgery

Neuro Cardio and Multispeciality Hospital

Biratnagar, Nepal

Email: dryamroka@yahoo.com

Date submitted: $14 / 11 / 2018$

Date accepted: $20 / 12 / 2018$

To access Nepal Journal of Neuroscience, Archives scan QR code:

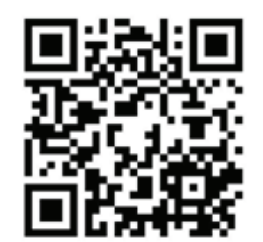

$\mathrm{L}$ earning from experience is inherent to animals and humans and when used in computer models it is termed as Machine learning (ML) which was coined by Arthur Samuel the pioneer of computer gaming and artificial intelligence in 1959. ${ }^{19}$ This field grew out during the search for artificial intelligence and initially was developed using neural networks, perceptrons, probabilistic reasoning and generalized linear models of statistics. ${ }^{18,20}$ ML uses "computational methods to
Nepal Journal of Neurosciences 15:3-7, 2018

\section{Brief Review of Machine Learning in Neurosurgery}

Learning from experience is inherent to animals and humans and when used in computer models it is termed as Machine learning (ML) which was coined by Arthur Samuel the pioneer of computer gaming and artificial intelligence in 1959. This field grew out during the search for artificial intelligence and initially was developed using neural networks, perceptrons, probabilistic reasoning and generalized linear models of statistics.ML works by either of the two methods, supervised learning or unsupervised learning. Search for "ML in neurosurgery" in Pubmed showed 308 results. There were 298 studies with abstracts, 5 clinical trials, 20 review articles and 168 articles in human studies. Of these around 113 articles were either studies of ML in other parts of the body like liver, stroke, EEG, pathology and Parkinsons disease or not involving ML and hence were excluded. Of the 55 remaining cases the majority were studies done in glioma followed by medical imaging in neurosurgery, radiotherapy, language and learning studies.ML will definitely replace many of the cumbersome physical data collection to infer and formulate ways to treat patients in the future. It can make the process of research accumulation, filter, find correlations between variables and help to make algorithms to manage and predict, that can save, time, money and speedup the recovery of the patient.

Key words: Artificial intelligence, Clustering, Data Mining, Predictive Modeling, Machine Learning, Machine Learning in Neurosurgery

"learn" information directly from data without relying on a predetermined equation as a model" and the computer learns without being programmed..$^{5,12} \mathrm{ML}$ searches through new data from prior built algorithms and then statistically calculates and forecasts the conclusion. It uses the process of data mining and predictive modeling to search and then predict the outcome. The common example is in daily life where the search engines give predictive search queries, related items and feeds advertisements based on 
Roka et al

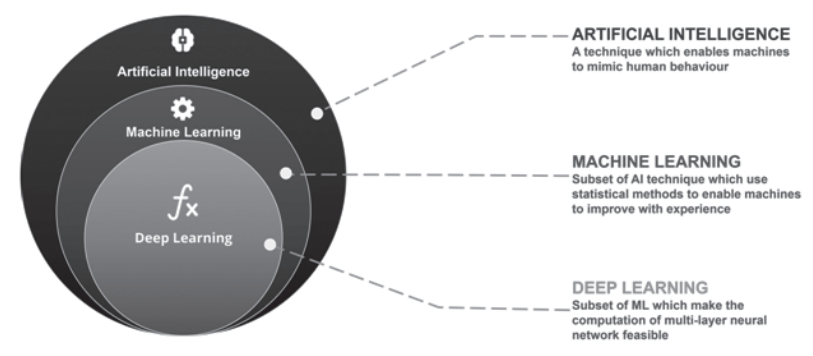

Figure1. Diagram showing the relation between artificial intelligence, Machine learning and deep Learning.6

the individual searches done. It can also be used to filter spam, security threats and building news feeds. ${ }^{5}$

Data mining: the process of going through large data and identify similar patterns to establish a relationship and solve problems through statistical analysis and foretell future trends.

Predictive modeling: the process using data mining and probability to predict outcomes based on multiple variables which are calculated using liner equation or complex neural network by sophisticated software.

ML is presently separate from Artificial intelligence (AI) and the former aims to use the available digitized information and use statistics and probability theory to solve problems and predict outcomes. ${ }^{12}$ Based on algorithms and computational data the machines learns from the data and the performance increases as the number of samples for study increases.

\section{How ML works:?}

ML works by either of the two methods, supervised learning or unsupervised learning (Figure 1). ${ }^{7}$

\section{Supervised learning:}

This method uses the knowledge of previous outcome based on a certain type of data and then uses this to predict outcome in a new set of data. A data analyst usually checks the input and output data and then the accuracy of the predictions to make an algorithm which will be then used for new data. Example: in cases with unruptured aneurysms, based on the available data on age, sex, site, size, comorbid conditions, habits and other variables ML can theoretically use this to predict the rupture of aneurysm in the next year.

This type uses classification and regression techniques to develop predictive models (Figure 2).

Classification techniques: predicts discrete responses and are used in medical imaging, credit scoring and speech recognition. Some of the algorithms used for classification are, support vector machine (SVM), k-nearest neighbor,

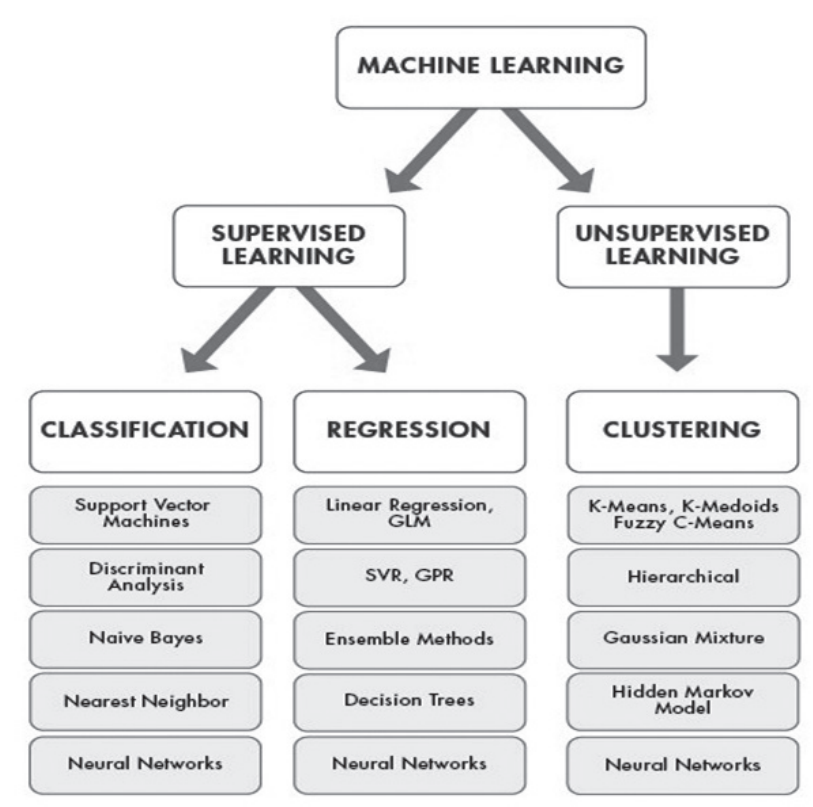

Figure 1: Figure showing ways data acquisition is done and used for supervised and unsupervised $M L{ }^{7}$

boosted and bagged decision trees, neural networks, Naïve Bayes, logistic regression, and discriminant analysis.

Regression techniques: these predict continuous responses with a data range or the response is a real number. Example: Blood pressure prediction based on variables like Age, Sex, Diet, and Weight is a type of regression study. ${ }^{21}$ Some common regression algorithms are linear model, stepwise regression, nonlinear model, regularization, neural networks, boosted and bagged decision trees, and adaptive neuro-fuzzy learning.

\section{Unsupervised learning: ${ }^{5,7}$}

This method finds concealed patterns and intrinsic structure in data using iterative approach called deep learning and draws inferences from input datasets without labeled responses (Figure 2).

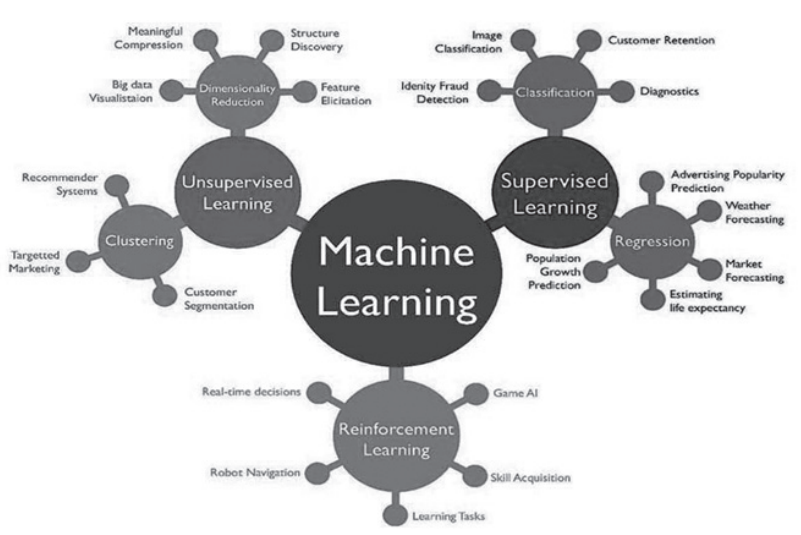

Figure 2: Figure showing ML and its uses. ${ }^{8}$ 
Machine learning in neurosurgery

\begin{tabular}{|c|c|c|}
\hline Specialty & Applications & Method \\
\hline Operation theatre & $\begin{array}{l}\text { Better mapping and preservation of } \\
\text { normal structures }\end{array}$ & $\begin{array}{l}\text { Registration of neuroanatomy based on } \\
\text { the functional geometry of fMRI } \\
\text { Matching a spectral embedding of the } \\
\text { functional connectivity patterns of two } \\
\text { fMRI volumes. }{ }^{13}\end{array}$ \\
\hline Neurooncology & $\begin{array}{l}\text { Better grading of tumors } \\
\text { Prognosticating outcome } \\
\text { Non invasive methods to diagnose }\end{array}$ & $\begin{array}{l}\text { Radiomic and machine } \\
\text { learningapproaches }\end{array}$ \\
\hline Epilepsy and Movement disorders & $\begin{array}{l}\text { Better localization of site of } \\
\text { lesionectomy } \\
\text { Better direct path/ } \\
\text { trajectory development }\end{array}$ & $\begin{array}{l}\text { Clinical, psychological and radiological } \\
\text { data mining } \\
\text { Supervised machine learning using } \\
\text { multimodal compared to unimodal } \\
\text { data accurately predicted postsurgical } \\
\text { outcome }^{15}\end{array}$ \\
\hline Neurotrauma & Predicting mortality after trauma & Studies in motorcycle riders ${ }^{11}$ \\
\hline Neuroimaging & $\begin{array}{l}\text { Better localization of the pathology } \\
\begin{array}{l}\text { Detecting the neural pathways/ } \\
\text { epileptic focus/uses of fMRI } \\
\text { navigation }\end{array} \\
\text { Anatomy of the aneurysm to predict } \\
\text { rupture }\end{array}$ & $\begin{array}{l}\text { Data mining from the massive amount of } \\
\text { data from CT scans, MRI and angiography } \\
\text { reports available } \\
\text { Geometric features of aneurysm } \\
\text { morphology useful non-invasive indicators } \\
\text { for the prediction of rupture risk }{ }^{24}\end{array}$ \\
\hline Stroke & $\begin{array}{l}\text { To detect and diagnose stroke } \\
\text { Treatment and prognosticate }\end{array}$ & $\begin{array}{l}\text { Multiple US FDAmobile apps available } \\
\text { Data from millions used to make algorithms } \\
\text { to predict stroke percentage in normal }\end{array}$ \\
\hline Spine disorders & $\begin{array}{l}\text { Outcome prediction in anterior } \\
\text { cervical discectomy } \\
\text { Prediction of mortality in spinal } \\
\text { metastases }\end{array}$ & $\begin{array}{l}\text { Artificial neural network, logistic } \\
\text { regression, support vector machine, and } \\
\text { random forest decision tree models } \\
\text { Creation of learning systems and } \\
\text { deployment of these systems as } \\
\text { accessible tools may significantly enhance } \\
\text { prognostication and management }^{10}\end{array}$ \\
\hline Neurosurgical care & \begin{tabular}{lr|} 
Preoperative planning, Intraoperative \\
guidance, Neurophysiological \\
monitoring, Neurosurgical outcome \\
prediction
\end{tabular} & $\begin{array}{l}\text { Increasing the efficiency and precision of } \\
\text { perioperative decision-making }{ }^{2,21}\end{array}$ \\
\hline NeuroRehabilitation & $\begin{array}{l}\text { Use of robotics/prosthesis } \\
\text { Remote controlled arms/limbs in } \\
\text { amputees } \\
\text { Vision recovery }\end{array}$ & Brain computer interface studies ${ }^{3}$ \\
\hline
\end{tabular}

Table 1. Showing the subspecialties in neurosciences and the ways ML can help. 


\section{Roka et al}

Clustering: the most common learning technique used is clustering. This type of ML is very complicated and uses millions of data to find correlation and subtle difference within the data and is commonly used in face recognition, speech to text prediction, natural language selection and gene sequence analysis. Common algorithms used are Gaussian mixture models, fuzzy c-means clustering, selforganizing maps, k-means and k-medoids, hierarchical clustering,hidden Markov models, and subtractive clustering.

\section{ML in Neurosurgery:}

Search for "ML in neurosurgery" in Pubmedshowed 308 results. There were 298 studies with abstracts, 5 clinical trials, 20 review articles and 168 articles in human studies. Of these around 113 articles were either studies of ML in other parts of the body like liver, stroke, EEG, pathology and Parkinsons disease or not involving ML and hence were excluded. Of the 55 remaining cases the majority were studies done in glioma followed by medical imaging in neurosurgery, radiotherapy, language and learning studies. Some of the interesting studies were face detection to diagnose Acromegaly, predict rupture of anterior communicating aneurysm, outcome in neurosurgical patients, prediction of trauma in motorcycleriders from Taiwan, impact of race on the discharge and length of hospitalization after brain tumor surgery, radiosurgery outcome in cerebral arteriovenous malformation, outcome after anterior cervical discectomy and a study of learning in patients with multiple concussions. ${ }^{1,2,4,11,14-17,22}$ Some of the common applications of ML in neurosurgery is given in Table 1.

\section{The Future of ML:}

With the use of more and more computers and smartphones in this world there has been a revolution even in the healthcare management systems. The recording or retrieving of data, its use to study the risk, develop algorithms to predict and then apply them to daily life has increased significantly over the last two decades. Presently there are so many mobile applications that help monitor the Oxygenation saturation, blood sugar level, health risk, exercise duration, wearable medical devices, calories spent and helps to determine the ideal diet based on individual Body Mass Index. Will this help to strengthen the clinician's clinical skills or weaken the decision making steps is yet to be determined as ML and AI are both yet to be developed fully. But if technology can help to diagnose, treat or manage the patient's disease better than it should definitely be accepted and implemented in medical practice.

By use of the millions of data collected like genetic, morphological, ethnic, molecular, radiological and pathological parameters ML can help treat neurosurgical disorders in an efficient and cost effective way. Data can be mined from the millions of unorganized articles, journals, meeting excerpts, textbooks using key words or algorithms and then used to provide an updated database which can be accessed to form organized disease specific data that can be used to predict, manage or formulate plans for best treatment. ML can overcome the problems and limitations with simple statistical data studies by increasing the number of variables studied and their overall relation with outcome. As given in the Table 1, there are many ML based algorithms that has helped in predicting outcome and decision making in many aspects of neurosurgery which has already proven its efficiency.

The question remains will ML be better than the experienced clinician to manage and treat diseases? Relying completely on stereotyped algorithms to predict the outcome in any one disease may still be questionable as there may be another variable that can influence the outcome which could be detected by the clinician and not ML.In those diseases like advanced stage gliomas, ML showing the outcome may not be of much use due to the fast growth and mortality of the disease itself. But in other diseases like risk of rupture of aneurysm, or the chances of stroke or cardiac arrest ML based prediction could benefit the population. So should we use ML in future in our clinics and operation theatre in all cases and will this lead to the weakening of the human ability to treat and prescribe medicine remains a topic of debate. The senior experience clinicians may find it difficult to use ML and probably their judgments would be better than ML but the juniors may resort to use of ML in their everyday practice which could cloud their clinical abilities. With the advancements in AI will the machines take over the clinical judgement ability of humans or shall we just be mere Neurosurgical Mechanics checking, inventing, feeding, building new algorithms and updating the newer Medical Machines is yet to be seen in future. Presently with ML still being developed it's my personal opinion that clinicians with their clinical judgments can manage patients better.

\section{Conclusion:}

ML will definitely replace many of the cumbersome physical data collection to infer and formulate ways to treat patients in the future. It can make the process of research accumulation, filter, find correlations between variables and help to make algorithms to manage and predict, that can save, time, money and speedup the recovery of the patient. The final decision as such will lie on the neurosurgeon to accept the overall results of ML and whether it will help the patient or not. The clinician's ability, experience, exposure and knowledge will be the deciding factor in patient 
management in future which could suggest that younger generation of physicians would probably use ML more often than their senior counterparts.

\section{References:}

1. Arvind V, Kim JS, Oermann EK, Kaji D, Cho SK. Predicting Surgical Complications in Adult Patients Undergoing Anterior Cervical Discectomy and Fusion Using Machine Learning. Neurospine 2018 Dec 17. doi: 10.14245/ns.1836248.124.

2. Celtikci E. A Systematic Review on Machine Learning in Neurosurgery: The Future of DecisionMaking in Patient Care. Turk Neurosurg 28(2): $167-$ 173, 2018

3. Gnanayutham P, Bloor C, Cockton G. Artificial Intelligence to enhance a Brain computer interface. HCI International 2003 proceedings, C. Stephanidis, Editor. 1397-401, 2003

4. Goswami R, Dufort P, Tartaglia MC, Green RE, Crawley A, et al. Frontotemporal correlates of impulsivity and machine learning in retired professional athletes with a history of multiple concussions. Brain Struct Funct 221 (4):1911-25, 2016

5. https://searchenterpriseai.techtarget.com/definition/ machine-learning-ML. accessed 22/12/2018

6. https://steemkr.com/science/@starlord6414/isartificial-intelligence-our-future-ch-3-deep-learningof-artificial-intelligence. Accessed 22 December 2018

7. https://www.mathworks.com/discovery/machinelearning.html, accessed 22/12/2018

8. https://www.techleer.com/articles/203-machinelearning-algorithm-backbone-of-emergingtechnologies/. Accessed 22/12/2018

9. Iv M, Zhou M, Shpanskaya K, Perreault S, Wang Z, Tranvinh E, et al. MR Imaging-Based Radiomic Signatures of Distinct Molecular Subgroups of Medulloblastoma. AJNR Am J Neuroradiol 2018 Dec 6. doi: 10.3174/ajnr.A5899

10. Karhade AV, Thio QCBS, Ogink PT, Shah AA, Bono CM, et al. Development of Machine Learning Algorithms for Prediction of 30-Day Mortality after Surgery for Spinal Metastasis Development. Neurosurgery 2018 Nov 23. doi: 10.1093/neuros/ nyy469.

11. Kuo PJ, Wu SC, Chien PC, Rau CS, Chen YC, Hsieh $\mathrm{HY}$, Hsieh CH. Derivation and validation of different machine-learning models in mortality prediction of trauma in motorcycle riders: a cross-sectional retrospective study in southern Taiwan. BMJ Open 5:8 (1):e018252, 2018
12. Langley, $P$. The changing science of machine learning. Machine Learning 82 (3): 275-279 (2011)

13. Langs, G, Golland, P, Tie, Y, Rigolo, L, \&Golby, A. J. Functional Geometry Alignment and Localization of Brain Areas. Advances in neural information processing systems 1: 1225-1233, 2010

14. Liu J, Chen Y, Lan L, Lin B, Chen W,et al. Prediction of rupture risk in anterior communicating artery aneurysms with a feed-forward artificial neural network. Eur Radiol 28 (8):3268-3275, 2018

15. Memarian N, Kim S, Dewar S, Engel J Jr, Staba RJ. Multimodal data and machine learning for surgery outcome prediction in complicated cases of mesial temporal lobe epilepsy. Comput Biol Med 64:6778,2015

16. Muhlestein WE, Akagi DS, Chotai S, Chambless LB. The Impact of Race on Discharge Disposition and Length of Hospitalization after Craniotomy for Brain Tumor. World Neurosurg 104:24-38, 2017

17. Oermann EK, Rubinsteyn A, Ding D, Mascitelli J, Starke RM, Bederson JB, Kano H, Lunsford LD, Sheehan JP, Hammerbacher J, Kondziolka D. Using a Machine Learning Approach to Predict Outcomes after Radiosurgery for Cerebral Arteriovenous Malformations. Sci Rep 9 (6): 21161, 2016

18. Russell, S. Norvig, Peter Artificial Intelligence: A Modern Approach (2nd ed.). Prentice Hall. ISBN 978-0137903955 (2003) [1995].

19. Samuel, A .Some Studies in Machine Learning Using the Game of Checkers. IBM Journal of Research and Development 3 (3): 210-229, 1959

20. Sarle, W S. Neural Networks and Statistical Models. Proceedings of the Nineteenth Annual SAS Users Group International Conference 4: 1-13, 1994

21. Schneider, A, Hommel, G, Blettner, M. Linear regression analysis: part 14 of a series on evaluation of scientific publications. Deutsches Arzteblatt international 107 (44): 776-82, 2010

22. Senders JT, Zaki MM, Karhade AV, Chang B, Gormley WB, Broekman ML, Smith TR, Arnaout O. An introduction and overview of machine learning in neurosurgical care. Acta Neurochir (Wien) 160 (1):29-38, 2018

23. Suchorska B, Schüller U, Biczok A, Lenski M, Albert NL, et al. Contrast enhancement is a prognostic factor in IDH1/2 mutant, but not in wild-type WHO grade II/III glioma as confirmed by machine learning. Eur J Cancer 107:15-27, 2018

24. Zhao X, Gold N, Fang Y, Xu S, Zhang Y, Liu J, Gupta A, Huang H. Vertebral artery fusiform aneurysm geometry in predicting rupture risk. R Soc Open Sci 5 (10): 180780, 2018 